\title{
MODELING OF POLLUTANT DISPERSION IN ATMOSPHERE BASED ON SOFTWARE COMPLEX "SKAT"
}

\author{
Aleksander V. Vorobiev ${ }^{1, *}$, Ekaterina M. Dutova ${ }^{1}$, and Andrei S. Latyshev ${ }^{1}$ \\ ${ }^{1}$ National Research Tomsk Polytechnic University, 634050 Tomsk, Russia
}

\begin{abstract}
The author of the article analyzes existing methods for calculation of pollutants ${ }^{6}$ dispersion in the atmosphere from a short continuous source under constant meteorological conditions. For this purpose the "SKAT" copyright software system elaborated by author was used. Furthermore, the author makes conclusions about advantages of using „Paskvilla method" for the calculation of gaseous substances" dispersion from sources of this type.
\end{abstract}

\section{Introduction}

The ecology of the regions is significantly affected by pollutants from the sources of natural and anthropogenic origin. Pollutant emissions enter various processes of air traffic, are released to the ground, spread in the hydrosphere contaminating the surface and groundwater.

Mathematical models and techniques adequately reflecting the dispersion of these substances in the atmosphere are required to describe and predict pollutant transport in the environment.

\section{Description of techniques}

In domestic practice, two techniques are currently most widely used, and they are recommended for calculation of air pollution with substances of industrial origin. One of the techniques developed under the guidance of $\mathrm{M}$. Berlyand laid the basis for the regulatory document OND-86 [1]. The second one is the technique for calculating the emission dispersion developed by F. Paskvill (Gaussian model), which is recommended by the World Meteorological Organization to assess the atmospheric pollution by industrial and power plants [2].

\subsection{The main concepts of the OND-86 technique}

The technique is based on the following assumptions [1]:

*Corresponding author: worob@tpu.ru 
- The pollutant dispersion in the atmosphere is affected by meteorological parameters (wind velocity and direction, temperature-stratification of the atmosphere and air temperature);

- The ground-level concentration of pollutants depends on the parameters of the emission source and composition of the air-gas mixture.

The calculation of spatial distribution of the pollutant concentration implies the calculation of the three basic parameters: the maximum ground-level concentration $C_{m}$; critical wind velocity $u_{m}$; the distance from the emission source to the point of maximum groundlevel concentration $x_{m}$ The highest concentration of the pollutant in the air $C_{m}$ for a given emission source appears in case of critical wind velocity and only at a certain source distance $x_{m}$.

The maximum ground-level concentration of harmful substances due to the release of the air-gas mixture from a single point source with a circular mouth is determined by the formula:

$$
C_{m}=\frac{A \cdot M \cdot F \cdot m \cdot n \cdot \eta}{H^{2} \cdot \sqrt[3]{V_{1} \cdot \Delta T}}
$$

where $C_{m}$ is measured in $\mathrm{mg} / \mathrm{m}^{3} ; A$ is a dimensionless coefficient that depends on the temperature-stratification of the atmosphere; $M$ is the mass of the pollutant emitted into the atmosphere per unit time, $\mathrm{g} / \mathrm{s} ; F$ is a dimensionless coefficient that takes into account the rate of pollutant sedimentation in the atmospheric air; $m, n$ are dimensionless coefficients that take into account the conditions of the air-gas mixture yield from the mouth of the emission source; $\eta$ is a dimensionless coefficient that takes into account the effect of the land topography; $H$ is the height of the emission source above the ground level, $\mathrm{m} ; V_{1}$ is the volumetric flow rate of the air-gas mixture, $\mathrm{m}^{3} / \mathrm{s} ; \Delta T=T_{g}-T_{a}$ is the differential temperature of the discharged air-gas mixture $T_{g}$ and the ambient air $T_{a},{ }^{\circ} \mathrm{C}$.

The OND-86 technique is currently approved at the state level and recommended for use to calculate the emission dispersion in the air while rationing and limiting maximum permissible emissions (MPE). However, this technique is difficult to use for scenario and forecast calculations under specific meteorological conditions for specific emission sources. The OND-86 technique does not fully takes into account the type of the underlying layer.

\subsection{The main concepts of the Paskvill's technique}

When pollutants are emitted into the atmosphere, a cloud is formed that drifts along with the atmospheric air with the wind. As a result of turbulent exchange with atmospheric air, the cloud expands over a distance and the concentration of pollutants in it changes. This leads to three-dimensional pollutants distribution described by the normal (Gaussian) law in the most general case.

According to this technique, the expression for the ground-level pollutant concentration from a short-time continuously emitting source (hereinafter SPC-source) under similar meteorological conditions is of the form [2]: 


$$
C(x)=\frac{Q}{\pi \cdot \sigma_{y} \cdot \sigma_{z} \cdot u} \cdot \exp \left[-\left(\frac{H_{e f f}^{2}}{2 \cdot \sigma_{z}^{2}}\right)\right] \cdot f_{F} \cdot f_{W},
$$

where $C(x)$ is the concentration of ground-level concentrations of harmful substances, $\mathrm{mg} / \mathrm{m}^{3} ; Q$ is the source power, $\mathrm{g} / \mathrm{s} ; H_{\text {eff }}$ is the effective emission height, $\mathrm{m} ; u$ is the wind velocity at the effective emission height, $\mathrm{m} / \mathrm{s} ; \sigma_{y}, \sigma_{z}$ are the dispersion coefficients of the Gaussian distribution (turbulent diffusion) in directions perpendicular to the wind, $\mathrm{m} ; f_{f}$ is the empirical depletion factor, which takes into account dry deposition; $f_{W}$ is the empirical depletion factor, which takes into account washout.

One of the main advantages of the Gaussian model is the possibility to account the impact of the following important factors on the pollutant distribution:

- Atmospheric stability;

- The type (roughness) of the underlying layer;

- Thermal rise of the stream due to overheating of the ejected air-gas mixture;

- Washing ability of precipitations and others.

\section{Software description}

We have developed a software package "SKAT" using the licensed Borland Turbo Delphi programming system to implement the algorithms of pollutant dispersion calculation according to the above techniques.

\section{Results of the calculations}

The software package "SCAT" was used to carry out comparative calculations of gaseous pollutant dispersion by the Gaussian model and the formulas of the OND-86 technique. A short-time continuously emitting source of $\left(\mathrm{SO}_{2}, \mathrm{NO}_{2}\right)$ flue gases was chosen as a test object.

The values of the input data taken for calculations correspond to the known [1] test calculation.

Fig. 1 shows the results of the calculation of the maximum values of ground-level concentrations $C_{m}$ of gaseous pollutants and the distances $x_{m}$ for the emission source where these values of the ground-level concentration are attained under various weather conditions.

Under favorable weather conditions (fig. 1, a), the modeling results of the distribution of the gaseous pollutant emission concentration along the plume centreline substantially depend on the technique (OND-86 or Paskvill's model) used for the calculation. The position of the point with the maximum ground-level concentration found by the OND-86 formulas virtually does not depend on meteorological parameter that contradicts the common sense and the data $[3,4]$.

On the contrary, under unfavorable weather conditions (fig. 1, b), the pollutant concentration distribution depends little on the calculation technique used. In particular, this concerns the location of the point with the maximum ground-level pollutant concentration. 


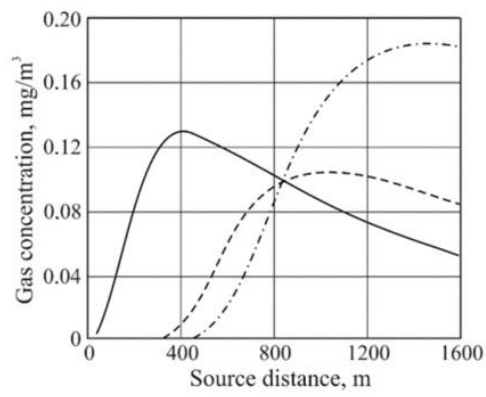

a)

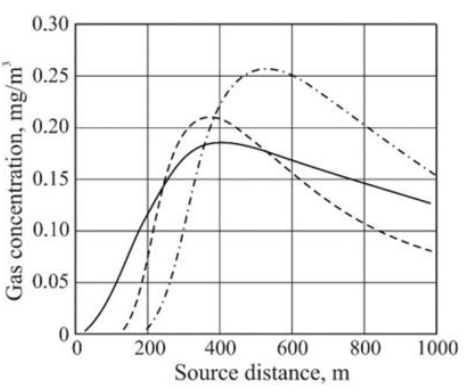

b)

Fig. 1. Distribution of the ground-level concentration of gaseous pollutants along the plume centreline at critical wind velocities corresponding to favorable (a) and unfavorable (b) weather conditions:- -- and $-\cdot-\cdot-$ - calculation by the Paskvill's model $\left(z_{0}=10 \mathrm{~cm}\right.$ and $\left.1 \mathrm{~cm}\right) ;-$ culation by the OND-86 technique.

\section{Conclusions}

The analysis of the techniques for calculation of gaseous substance dispersion for a shorttime continuously emitting source suggests that under stable meteorological factors the Paskvill's model is preferable as it takes into account a wider range of the key parameters which adequately describe the local weather conditions and the type of the underlying surface.

The developed software package "SKAT" can be used for calculating the pollutant dispersion in the atmosphere to solve the monitoring problems of air pollutant emission for currently emitting sources.

\section{References}

1. OND-86. The method of calculating the concentration of harmful substances emitted by industrial enterprises in the atmospheric air (Gidrometeoizdat, Leningrad, 1987)

2. Method of development of standards for maximum permissible emissions of radioactive substances in the atmospheric air (DV, 2010)

3. M. R. Beychock, Fundamentals of Stack Gas Dispersion (Author-Published, 2005)

4. S. Arya, Pal Air Pollution Meteorology and Dispersion (Oxford University Press, Oxford, 1998)

5. Schnelle, B. Karl, and P. R. Dey, Atmospheric Dispersion Modeling Compliance Guide (McGraw-Hill Professional, 1999)

6. D.B. Turner, Workbook of Atmospheric Dispersion Estimates: An Introduction to Dispersion Modeling (CRC Press, 1994)

7. K. Wark, C.F. Warner, and W. T. Davis, Air Pollution: Its Origin and Control (PreticeHall, 1998)

8. A. Riddle, D. Carruthers, A. Sharpe, C. McHugh, J. Stocker, Atm. Env. 38 (2004)

9. R. Barrat, Atmospheric Dispersion Modelling (Earthscan Publications, 2001)

10. A. Daly, P. Zannetti. 2007 Air Pollution Modeling (The Arab School for Science and Technology, 2007) 